\title{
Las Metas del Milenio (servicios de saneamiento y agua potable) de 2000 a 2010 en Cozumel y Playa del Carmen, Quintana Roo, México
}

\author{
Justo Rojas López* \\ Centro de Estudios Sociales y Ambientales del Caribe \\ Oscar Frausto Martínez \\ Universidad de Quintana Roo, Unidad Académica Cozumel \\ Javier Tun Chim \\ Observatorio de la Vivienda de Guanajuato \\ Thomas Ihl \\ Centro de Investigaciones en Geografía Ambiental, UNAM
}

Resumen

Entre las Metas del Milenio suscritas en el año 2000 está reducir la falta de acceso al agua potable y el saneamiento en los hogares pobres, considerados fundamentales para mantener la subsistencia de la raza humana. Las ciudades del norte de Quintana Roo, México, han enfrentado en los últimos 20 años altos crecimientos poblacionales, que retan el suministro de estos servicios urbanos. El presente trabajo mide la provisión del agua potable y del saneamiento urbano para los últimos diez años en Cozumel y en Playa del Carmen, junto con la intensidad de las presiones demográficas sufridas, a fin de verificar si han sido capaces de proveer estos servicios básicos a sus habitantes y evaluar si existen avances en el contexto de las Metas del Milenio en el nivel local. Los resultados muestran que al inicio del periodo de estudio Cozumel consigue los mejores resultados, en la mayoría de los casos incidencias sobre $90 \%$, sin embargo, Playa del Carmen emprende un crecimiento acelerado hasta igualar o mejorar la cobertura de Cozumel, la cual incluso empeora su provisión de estos servicios.

\section{Palabras Clave}

Agua, saneamiento, Metas del Milenio, Riviera Maya.

*Correo electrónico: rojasjusto@gmail.com 


\title{
Millennium Development Goals (sanitation and drinking water) from 2000 to 2010 in Cozumel and Playa del Carmen, Quintana Roo, Mexico
}

\author{
Justo Rojas López* \\ Centro de Estudios Sociales y Ambientales del Caribe \\ Oscar Frausto Martínez \\ Universidad de Quintana Roo, Unidad Académica Cozumel \\ Javier Tun Chim \\ Observatorio de la Vivienda de Guanajuato \\ Thomas Ihl \\ Centro de Investigaciones en Geografía Ambiental, UNAM
}

\section{Abstract}

Among the Millennium Development Goals signed in 2000 is a plan to reduce the lack of access to clean water and to improve poor sanitation in households at the poverty level. This is essential in order to maintain the survival of the human race. Northern cities in Quintana Roo, Mexico, have faced high population growth in the last 20 years challenging the provision of these urban public health services. This study quantifies the provision of drinking water and urban sanitation over the last ten years in Cozumel and Playa del Carmen and the demographic pressures suffered. All this is in order to verify if the basic specific public services have been provided to its inhabitants and also to assess whether or not there is progress at the local level in the context of the Millennium Development Goals. Results show that at the beginning of the ten-year-study period, Cozumel got the best results, in most cases with over a 90\% success rate. However, Playa del Carmen is undertaking rapid growth to meet or exceed the coverage of Cozumel and will overtake Cozumel in providing public services.

\section{KEY WORDS}

Water, sanitation, Millennium Goals, Riviera Maya.

*E-mail: rojasjusto@gmail.com 


\section{Introducción}

El acceso al agua potable y a los servicios de saneamiento del hogar vinculados al agua integra una faceta de la satisfacción de necesidades esenciales para la vida (Boltvinik, 1999: 314; CIAMA, 1992: 2; ONU, 2010b: 3). Además, su presencia se estima fundamental para evitar ciertos desastres naturales relacionados con inundaciones 0 huracanes, desastres sociales (Lavell, 2000: 8), y los causados por enfermedades contagiosas (Rojas López, 2010: 75). Es decir, la provisión de estos servicios tiene que ver con casi cualquier consideración de bienestar humano.

Por ello, en la Declaración del Milenio de las Naciones Unidas del año 2000 (ONU, 2000: 7) y en la Cumbre Mundial sobre el Desarrollo Sostenible, celebrada en Johannesburgo en 2002 (ONU, 2002: 11), se establece como compromiso de los gobiernos firmantes, entre ellos el de México: "reducir a la mitad para el año 2015 el porcentaje de personas que no tiene un acceso sostenible al agua potable y a servicios básicos de saneamiento". Se entiende como servicios básicos de saneamiento el drenaje conectado a la red pública y a fosa séptica, así como el acceso a servicio sanitario con conexión de agua (ONU-Hábitat/Sedesol, 2012) (objetivo 7, meta 10 y punto 18).

Considerando lo anterior, el presente trabajo, que surge de la recapitulación del monitoreo que el Observatorio Urbano Riviera Maya de la Universidad de Quintana Roo y el Centro de Estudios Sociales y Ambientales del Caribe han venido realizando desde 2004 en diferentes proyectos, toma de la lista de indicadores del Programa de las Naciones Unidas para los Asentamientos Humanos (ONU-Hábitat) y la Secretaría de Desarrollo Social (Sedesol) -los cuales son la adaptación oficial para México de los indicadores para la evaluación de los avances de las Metas del Milenio- aquellos vinculados a estos servicios, y se realiza un análisis transversal que abarca diez años (2000, 2005 y 2010) para Cozumel y Playa del Carmen en la Riviera Maya en Quintana Roo, con el que se miden los cambios en su distribución en el nivel de ciudad y, en algunos casos, dentro de ellas. Así, este análisis puede sumarse directamente, desde una perspectiva regional, a la evaluación de los avances que el país se comprometió a efectuar en las Metas del Milenio.

El análisis se complementa con una comparación de las presiones demográficas que tienen ambas ciudades para la provisión de los servicios mencionados, ya que, en el contexto regional, estas dos ciudades han enfrentado en los últimos 15 años elevados crecimientos poblacionales, los cuales se han ubicado entre los más altos 
del país (Conapo, 2004: 1; INEGI, 2010: 1), en especial Playa del Carmen, que ha sido considerada una de la ciudades con mayor crecimiento poblacional en los últimos diez años. Tal crecimiento poblacional, principalmente causado por un saldo neto migratorio positivo, habría generado fuerte presión en la región hacia los intentos del estado por proveer estos servicios básicos.

\section{Metodología}

Con base en las metodologías de la Agenda Hábitat-Sedesol (2002: 58), se calcularon los indicadores "Acceso al agua segura", "Conexiones domiciliarias" y "Saneamiento mejorado" para Cozumel y Playa del Carmen, Quintana Roo, en los años 2000, 2005 y 2010. Cada uno tiene particularidades que se explican a continuación.

"Acceso al agua segura" (AAS) considera las viviendas que tienen acceso al agua potable, excluyendo únicamente a aquellas que lo hacen por medio de pipas. Es obtenido para las ciudades de Cozumel y Playa del Carmen en los años 2000 y 2005, para los municipios correspondientes de Cozumel y Solidaridad en 2010, y para las áreas geoestadísticas básicas (AGEB) de cada una de las ciudades en cuestión para el 2000.

"Saneamiento mejorado" (SM) incluye a las viviendas con servicios sanitarios exclusivos, conexión de agua a la red pública o admisión manual de agua y drenaje conectado a la red pública o a fosa séptica, y es obtenido en el nivel de ciudad para los años 2000 y 2005, a la vez que en el nivel municipal en 2010.

"Conexiones domiciliarias" (COD) tiene que ver con las viviendas que disponen de energía eléctrica, agua potable y drenaje conectado a la red pública. Es obtenido en los mismos niveles de desagregación que el indicador de AAS, es decir, en el nivel de ciudad (2000 y 2005) y municipal (2010), así como para las AGEB (2000).

Además de estos indicadores, el trabajo también empleó de la misma lista de ONUHábitat el de "Crecimiento de la población urbana”, que da señas de la situación de la presión población sobre estos servicios. Este indicador utiliza el estadístico crecimiento medio anual, el cual representa un porcentaje promedio de los crecimientos en un lapso determiando. Cabe anotar que para el año 2000 se tomó como referencia el crecimiento de las ciudades de 1995 a 2000; para 2005, el de 2000 a ese año, en 
tanto que para 2010 se consideró el crecimiento poblacional de los municipios ${ }^{1}$ de 2005 a 2010.

En los casos donde fue posible, los resultados de los indicadores del agua y el saneamiento del hogar se introdujeron en un sistema de información geográfica para generar mapas que describen su distribución territorial.

\section{Resultados}

A continuación se muestran los principales resultados de los indicadores propuestos. Se divide la disertación por cada uno de ellos, para después, en conjunto, contrastarlos con el indicador "Crecimiento de la población urbana".

\section{Acceso al agua segura}

CUADRo 1. Agua segura en la Riviera Maya 2000-2010

\begin{tabular}{lccc} 
Presencia de Agua segura (\%) & $\mathbf{2 0 0 0}$ & $\mathbf{2 0 0 5}$ & $\mathbf{2 0 1 0}$ \\
Cozumel (Cozumel) & 95.60 & 99.54 & 96.06 \\
Playa del Carmen (Solidaridad) & 92.61 & 97.94 & 98.91 \\
$\begin{array}{l}\text { Crecimiento medio anual } \\
\text { del Agua segura (\%) }\end{array}$ & $\mathbf{2 0 0 0 - 2 0 0 5}$ & $\mathbf{2 0 0 5 - 2 0 1 0}$ \\
Cozumel (Cozumel) & 0.81 & -0.71 \\
Playa del Carmen (Solidaridad) & 1.13 & 0.20 \\
\hline
\end{tabular}

Fuente: Elaboración propia con base en el INEGI (2000, 2005 y 2010).

Los resultados (cuadro 1) manifiestan, por un lado, que el indicador "Acceso al agua segura" para ambas ciudades ha presentado de 2000 a 2010, tal como lo reflejan los análisis nacionales (Conapo, 2005: 22; Coneval, 20011: 8), buenos resultados, niveles de provisión sobre $90 \%$ para cada año; y, por el otro, un crecimiento en su provisión en las dos ciudades entre 2000 y 2010. Auxiliarmente, existen algunos datos que destacar: al iniciar la década, Cozumel tiene el mayor porcentaje

${ }^{1}$ Esto porque hasta la fecha en que se elaboró el presente documento el Instituto Nacional de Estadística y Geografía (INEGI) no tenía disponibles los datos para su cálculo en el nivel de localidades. 
de provisión por casi 3 \% (2.99\%) sobre Playa del Carmen, pero a la mitad de la década esta diferencia, aunque disminuye $(1.96 \%$ ), se mantiene a favor de Cozumel. Sin embargo, al finalizar la década las cosas se revierten y Playa del Carmen tiene el mayor porcentaje de cobertura sobre Cozumel por $2.85 \%$. Este cambio en los niveles de provisión de las ciudades se debe a un ritmo de crecimiento diferenciado entre ambas. Así, aunque entre 2000 y 2005 las dos ciudades incrementan su abasto, Playa del Carmen exhibe un ritmo más acelerado (1.13\% anual y $5.33 \%$ global) que Cozumel ( $0.81 \%$ anual y $3.94 \%$ global), en tanto que, entre 2005 y 2010 , a pesar de que ambas desaceleran su crecimiento, Playa del Carmen aún crece ( $0.20 \%$ anual y $0.97 \%$ global), en contraste con Cozumel, que disminuye su provisión (-0.71 \% anual y -3.47\% global). Estos resultados sugieren que, si bien pueden calificarse de manera general como buenos, en realidad en los últimos años ambas ciudades sufren una caída en la capacidad de provisión de estos servicios, al grado de que en Cozumel desciende a valores cercanos a los que mostraba al principio.

Ahora, al apreciar por medio de las AGEB (figura 1 A, B, C y D) la configuración interna de este indicador en las dos ciudades para 2000 y 2010, es clara la desigualdad de acceso entre diferentes zonas de la ciudad en los dos años.

Lo primero y más evidente que resulta de este indicador por AGEB en el 2000 (figura 1A y B) es que en ambas ciudades existen áreas con niveles inferiores a los que muestran en promedio, es decir, se presentan distribuciones heterogéneas de este indicador, llegando en algunos casos a observarse áreas con porcentajes debajo de $75 \%$ de provisión de estos servicios (dos en Cozumel y seis en Playa del Carmen). Un segundo dato a destacar es que las AGEB con estos niveles por debajo de los promedios se encuentran en su mayoría en las periferias de las ciudades, o sea, en las secciones más alejadas de los centros de las ciudades ubicadas frente a la costa.

En el análisis de las ciudades en 2000, Playa del Carmen muestra que, al oeste, en la sección que corresponde a las colonias que crecieron después de la zona central y concentraron la primera zona de vivienda popular, está la mayor cantidad de AGEB (siete) con porcentajes de provisión de agua segura inferiores al promedio de la ciudad, por lo cual muy probablemente representaban una región con problemas de acceso al agua segura, a diferencia del resto de la ciudad. Por otra parte, se distinguen tres AGEB en la zona norte de la ciudad con porcentajes por debajo del promedio, que corresponden a la colonia Colosio, la cual se originó en los años noventa debido a una invasión de personas a terrenos privados. 


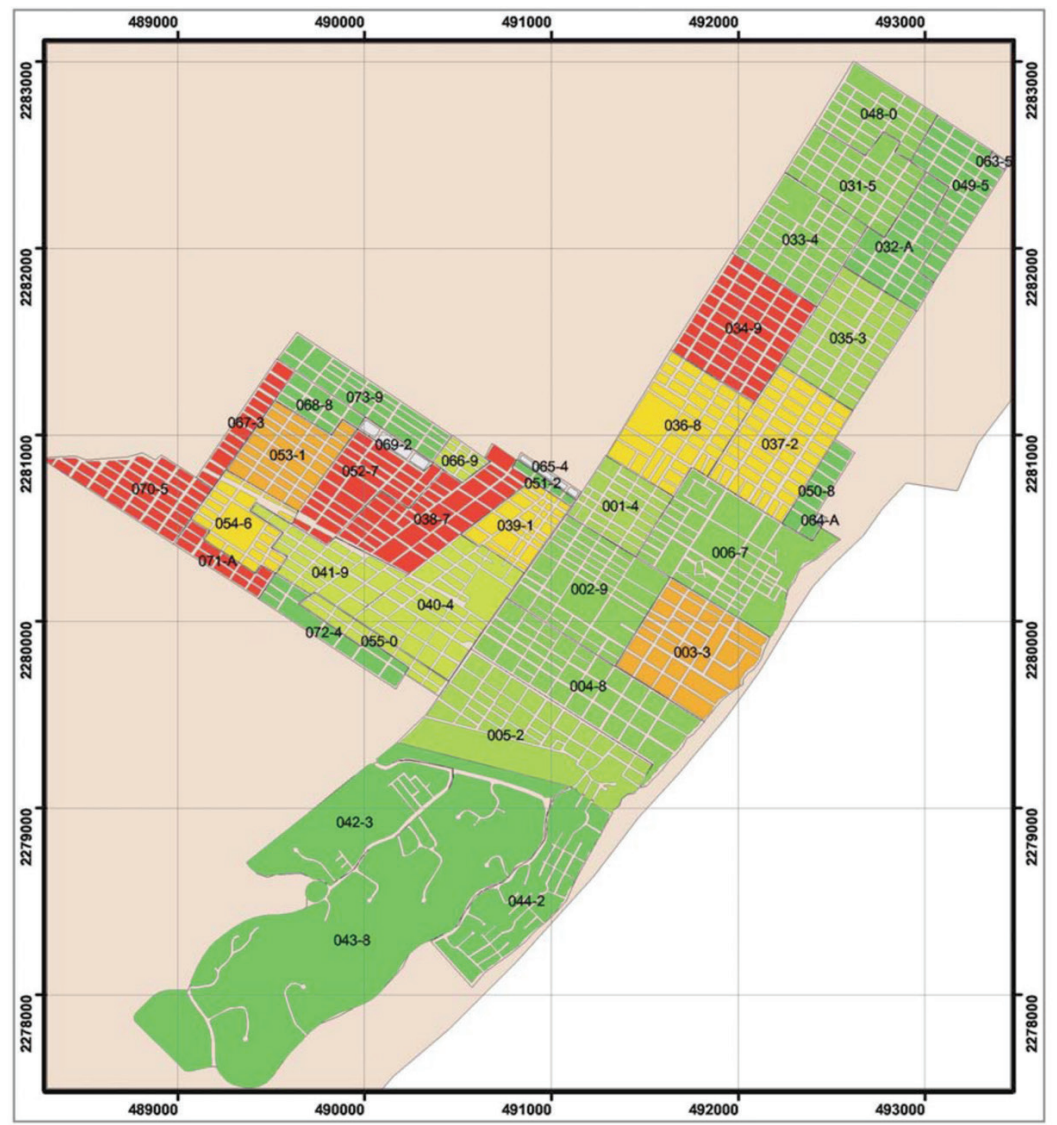

\section{Indicador Clave 4: Acceso a agua potable}

Porcentaje de hogares con acceso a un suministro mejorado de agu

Leyenda:

Acceso a agua potable [en \%]:

\begin{tabular}{|l|l|}
\hline no data & $92,05-94,12$ \\
\hline $0,01-75,45$ & $94,13-98,15$ \\
\hline $75,46-85,61$ & $98,16-99,21$ \\
\hline $85,62-92,04$ & $99,22-100,00$ \\
\hline
\end{tabular}

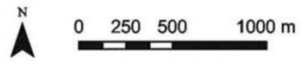

Proyección: NAD 1927 UTM Zone $16 \mathrm{~N}$ Fuente: INEGI 2000 y SEDESOL 2000

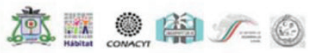

1-A. Playa del Carmen, 2000 


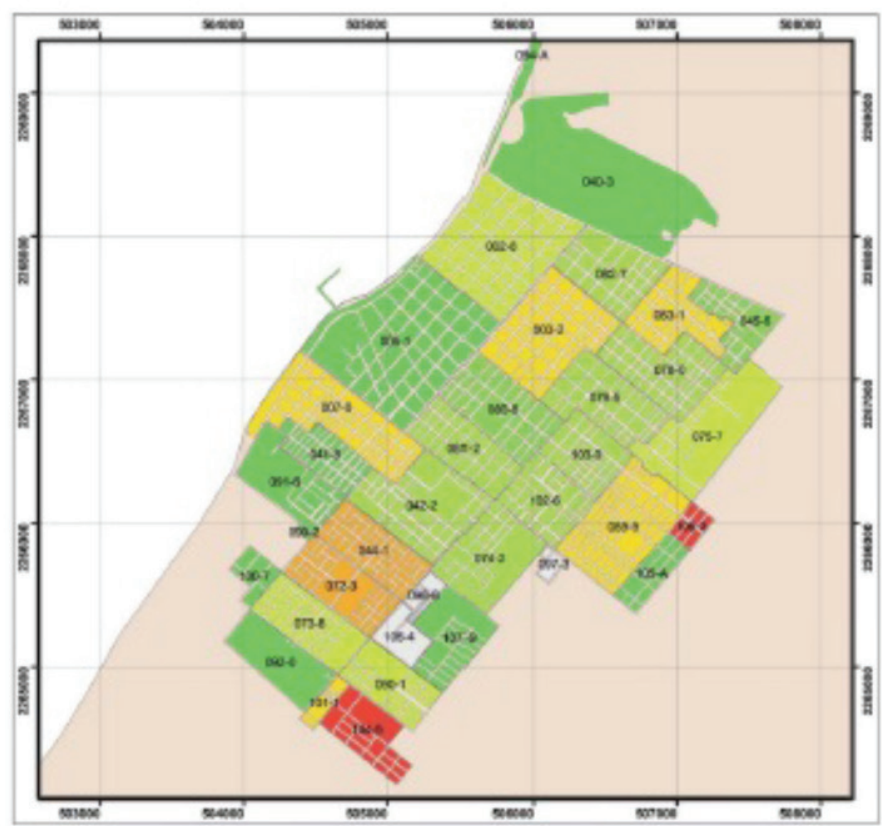

Indicador Clave 4. Acceso a agua potable

Ponceniaje de hogares con scceso o suministor mejo ods de squa.

Acceso a agua potable [en $\%$ ]:

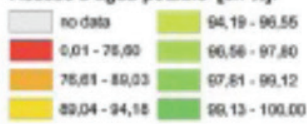

MGEA

Musieplo Conume

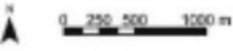

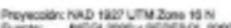

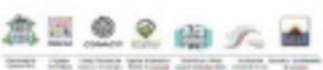

1-B Cozumel, 2000

FIGURA 1-B. Distribución del Agua segura por áreas geoestadísticas básicas (AGEB) 


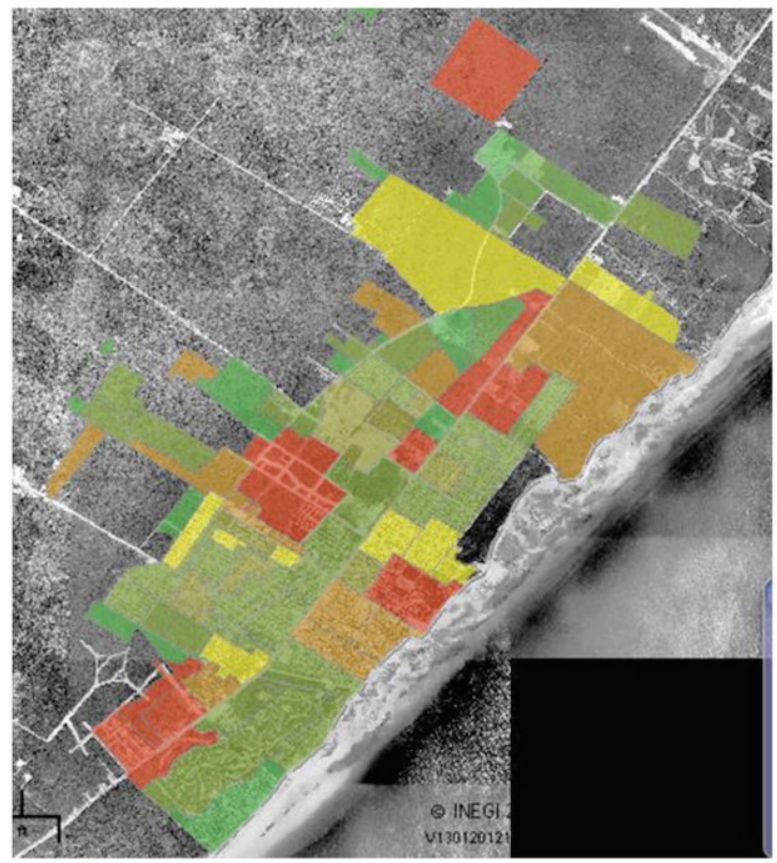

Indicador clave 4. Acceso a agua potable por AGEB 2010 de la zona urbana de Playa del Carmen

\begin{tabular}{|c|c|c|}
\hline \multicolumn{3}{|c|}{ Acceso a agua segura } \\
\hline ? & 99.210 & $<=100$ \\
\hline ? & 98.150 & $<=99.21$ \\
\hline ? & 94.120 & $c=98.15$ \\
\hline ? & 92.040 & $<=94.12$ \\
\hline.$>$ & 85.61 & $<=92.04$ \\
\hline ? & 75.450 & $<=85.61$ \\
\hline$P=$ & 42.860 & $c=75.45$ \\
\hline
\end{tabular}

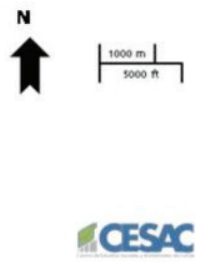

1-C. Playa del Carmen, 2010 


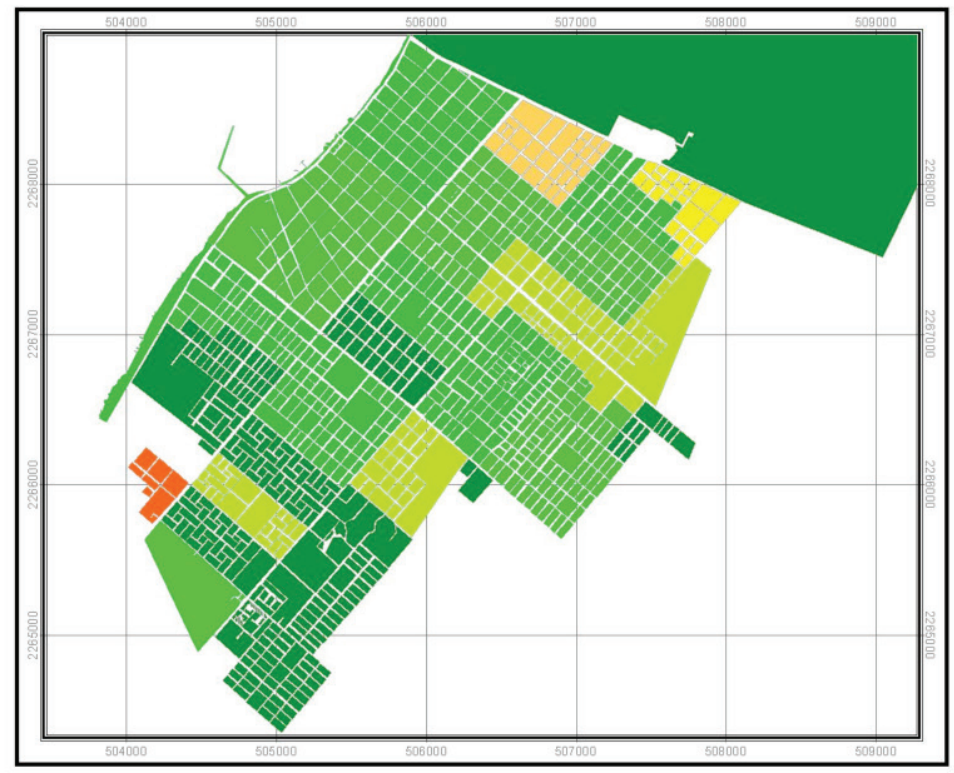

Indicador clave 4. Acceso a agua potable por AGEB 2010 de la zona urbana de Cozumel

Acceso a agua segura

\begin{tabular}{|l|l|l|}
\hline $0.01-76.6$ & & $94.19-96.55$ \\
\hline $76.61-89.03$ & & $96.56-97.8$ \\
\hline $89.04-94.18$ & & $97.81-99.12$ \\
\hline & & $99.13-100$ \\
\hline
\end{tabular}

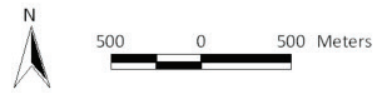

ICESAC

1-D. Cozumel, 2010

FIGURA 1-D. Distribución del Agua segura por áreas geoestadísticas básicas (AGEB) 
En Cozumel, la parte sur de la mancha urbana en las colonias San Miguel, que surgieron a principios de los noventa principalmente por vivienda popular, y la parte este en las colonias San Gervasio-Chen Tuk, formadas a finales de los noventa también por vivienda popular, presentan las mayores concentraciones de AGEB con porcentajes de provisión de agua segura por debajo del promedio de la ciudad. Una particularidad de Cozumel es que se observan otras AGEB (tres) con los mencionados porcentajes fuera de las zonas descritas, lo cual significaría que además de las regiones que concentran estos resultados hay deficiencias dispersas por la ciudad.

En 2010 (Figura 1C y D), la perspectiva cambia en las dos ciudades. Para Playa del Carmen, aunado a su notorio crecimiento de la mancha urbana con base en el año 2000, se encuentra que la zona costera en lo que se considera el centro de la ciudad tiene valores por debajo del promedio e incluso de entre los más bajos de la ciudad. Los sectores al oeste de la mancha urbana también tienen AGEB con los mayores rangos de carencia, que se ubican en las colonias Ejidales y al este del Pedregal, en la parte norte en las AGEB al este de la colonia Luis Donaldo Colosio y al oeste de Bosque Real y Mundo Hábitat, todas colonias de más de diez años de existencia; además, la AGEB que comprende las colonias Guadalupana, El Petén y Villas Riviera, de reciente creación (aproximadamente tres años). En la parte sur, en las AGEB donde se localiza la colonia Campestre y sus alrededores (que se originaron en los últimos cuatro años), también se advierten porcentajes de entre los más bajos de la ciudad. Así, en Playa del Carmen gran parte de las AGEB que en 2010 mostraron los niveles de carencia más altos son colonias que ya existían en 2000, por lo que en realidad sus condiciones empeoraron o por lo menos permanecieron igual, esto último en el caso de las que se encuentran en las colonias Ejidales.

En Cozumel, la situación es distinta de la de Playa del Carmen, ya que la mayoría de sus colonias se ubica en los porcentajes medios y altos de acceso a este servicio. Sin embargo, los porcentajes de mayor carencia están al norte de la ciudad, en la parte norte de la colonia 10 de Abril y Emiliano Zapata, así como en la sección sur de la colonia Colonos Cuzamil (colonias con más de diez años de existencia). 


\section{Saneamiento mejorado}

CUADRO 2. Saneamiento mejorado en la Riviera Maya 2000-2010

\begin{tabular}{lccc} 
Presencia Saneamiento mejorado (\%) & $\mathbf{2 0 0 0}$ & $\mathbf{2 0 0 5}$ & $\mathbf{2 0 1 0}$ \\
Cozumel (Cozumel) & 87.42 & 97.79 & 97.21 \\
$\begin{array}{l}\text { Playa del Carmen (Solidaridad) } \\
\text { Crecimiento medio anual del Saneamiento }\end{array}$ & 45.37 & 60.62 & 97.77 \\
$\begin{array}{l}\text { mejorado (\%) } \\
\text { Cozumel (Cozumel) }\end{array}$ & $\mathbf{2 0 0 0 - 2 0 0 5}$ & $\mathbf{2 0 0 5 - 2 0 1 0}$ \\
Playa del Carmen (Solidaridad) & & 2.27 & -0.12 \\
\hline
\end{tabular}

Fuente: Elaboración propia con base en el INEGI (2000, 2005 y 2010).

Los resultados respecto a este indicador (cuadro 2) señalan, primero, que las ciudades inician con dos niveles de provisión de este bien muy disímiles y, segundo, que ambos mejoraron en términos generales a lo largo del tiempo. Para Playa del Carmen, los resultados al principio pueden considerarse malos, ya que menos de la mitad de sus habitantes tenía acceso al saneamiento mejorado, mientras que para Cozumel pueden juzgarse buenos, al estar apenas debajo del $10 \%$ de habitantes con deficiencia en este indicador. Adicionalmente, en ambas ciudades el indicador ha mostrado, al principio del decenio, resultados de provisión inferiores a los exhibidos por el indicador "Acceso al agua segura"; además, en ese mismo momento Playa del Carmen tenía niveles inferiores a 50 \% (45.37\%) de acceso a saneamiento mejorado y Cozumel inferiores a $90 \%$ (87.42\%), esto es, en el año 2000 su nivel de provisión en Cozumel era muy superior al de Playa del Carmen por casi el doble (42 puntos porcentuales).

Empero, también es claro que esta diferencia, similar a lo sucedido con el indicador anterior, se fue reduciendo hacia el final de la década. En 2005 la distancia entre las dos ciudades es de $37.17 \%$, y en 2010 Playa del Carmen sobrepasa a Cozumel por algunas décimas (56) de punto porcentual. Esta reducción de la diferencia se explica por los ritmos de crecimiento de este indicador entre las ciudades: entre 2000 y 2005 Playa del Carmen incrementa su abastecimiento $15.25 \%$, que representa un crecimiento medio anual de $5.97 \%$, en tanto que en el mismo lustro 
Cozumel lo eleva $10.37 \%$, lo cual significa un crecimiento medio anual de $2.27 \%$, es decir, su crecimiento es casi de la mitad del de Playa del Carmen. En la segunda mitad de la década, Playa del Carmen aumenta su porcentaje $37.15 \%$, lo que en términos de su crecimiento medio anual representa $10.03 \%$, en cambio, Cozumel disminuye su cobertura $0.58 \%$, que en crecimiento medio anual significa $-0.12 \%$.

Este comportamiento revela que Cozumel, tal como ocurrió en el indicador "Acceso al agua segura”, tiene una disminución de su capacidad de provisión de estos servicios; en contraste, en Playa del Carmen esa capacidad se ha incrementado notablemente sobre todo en los últimos años.

\section{Conexiones domiciliarias}

CuAdRo 3. Conexiones domiciliarias en la Riviera Maya 2000-2010

\begin{tabular}{lccc}
$\begin{array}{l}\text { Presencia Conexiones } \\
\text { domiciliarias (\%) }\end{array}$ & $\mathbf{2 0 0 0}$ & $\mathbf{2 0 0 5}$ & $\mathbf{2 0 1 0}$ \\
Cozumel (Cozumel) & 85.21 & 93.47 & 94.29 \\
$\begin{array}{l}\text { Playa del Carmen (Solidaridad) } \\
\text { Crecimiento medio anual de } \\
\text { Conexiones domiciliarias (\%) }\end{array}$ & 44.86 & 57.48 & 86.91 \\
$\begin{array}{l}\text { Cozumel (Cozumel) } \\
\text { Playa del Carmen (Solidaridad) }\end{array}$ & $\mathbf{2 0 0 0 - 2 0 0 5}$ & $\mathbf{2 0 0 5 - 2 0 1 0}$ \\
\hline
\end{tabular}

Fuente: Elaboración propia con base en el INEGI (2000, 2005 y 2010).

Los datos en torno a este indicador (cuadro 3) exponen que al inicio de la década ambas ciudades presentan resultados similares al indicador anterior: Playa del Carmen con niveles inferiores a $50 \%$ de provisión de este servicio y Cozumel con porcentajes por debajo de $90 \%$. No obstante, estos resultados son ligeramente inferiores a los del indicador previo. Además, como sucedió en los dos indicadores pasados, este servicio aumentó su cobertura en las dos ciudades en los siguientes años, aunque no al mismo ritmo. Playa del Carmen casi la duplica y Cozumel la incrementa cerca de $10 \%$. Para Playa del Carmen el indicador eleva su porcentaje de 2000 a 2005 un $12.62 \%$, lo que representa un crecimiento medio anual de $5.08 \%$; 
en tanto que entre 2005 y 2010 su aumento es de $29.43 \%$, con un crecimiento medio anual de $8.62 \%$; esto significa que en la segunda parte de la década sobreviene el mayor crecimiento de este servicio en la ciudad. En cambio, en Cozumel, entre 2000 y 2005 este indicador se eleva $8.26 \%$, que en términos de su crecimiento promedio anual significa $1.87 \%$, y para la segunda parte de la década el incremento fue de tan sólo $0.86 \%$, lo cual en la media anual constituye $0.17 \%$. Esto indica que, a diferencia de Playa del Carmen, que crece en mayor media de 2005 a 2010, Cozumel tiene su mayor crecimiento de abastecimiento en la primera parte de la década. Es claro además que al principio de la década Cozumel tiene una provisión que casi dobla (40.35 puntos porcentuales más) a la de Playa del Carmen, pero al pasar el tiempo la distancia se reduce entre ambas ciudades hasta el punto de que en 2010 la diferencia es de menos de diez puntos porcentuales (7.38).

De acuerdo con estos resultados, en el 2000 Playa del Carmen tenía una provisión muy baja del servicio -menos de la mitad de sus habitantes-, la cual fue aumentando lentamente durante los primeros cinco años, pero en la segunda mitad su crecimiento fue acelerado, al grado de cubrir en 2010 a casi siete de cada ocho habitantes. Por su parte, en Cozumel se nota que al iniciar la década se tenía un provisión alta -seis de cada siete habitantes-, que se elevó de manera importante en el primer quinquenio, hasta sobrepasar $90 \%$ de su cobertura, pero fue disminuyendo el ritmo de provisión hasta sólo incrementar poco menos de un punto porcentual durante los últimos cinco años de la década.

En cuanto a la distribución interna de este indicador en ambas ciudades por medio de las AGEB (figura 2A, B, C y D), se observa que, con base en la información del año 2000, en Playa del Carmen (figura 2-A) dos zonas concentran las AGEB con mayores deficiencias de este indicador: la primera al norte de la ciudad, en la colonia Colosio, donde seis de sus AGEB tienen la mayor carencia (entre 0.01 y $13 \%$ ) de tenencia de este servicio y dos más se encuentran por debajo de $34.56 \%$. En tanto que la segunda zona corresponde al este de la ciudad, en las colonias posteriores a la carretera federal, en las que seis de sus AGEB muestran porcentajes de provisión entre 13.17 y $26.14 \%$ (menores a 1 de 3 ocupantes con este servicio); asimismo, cinco más se encuentran por debajo de $57.33 \%$ de habitantes con este servicio. De tal suerte, es notorio que en esta ciudad las áreas con problemas estaban altamente concentradas en dos zonas, caracterizadas por ser las de más reciente creación y las que mayoritariamente se dispusieron para la vivienda de alta densidad. 


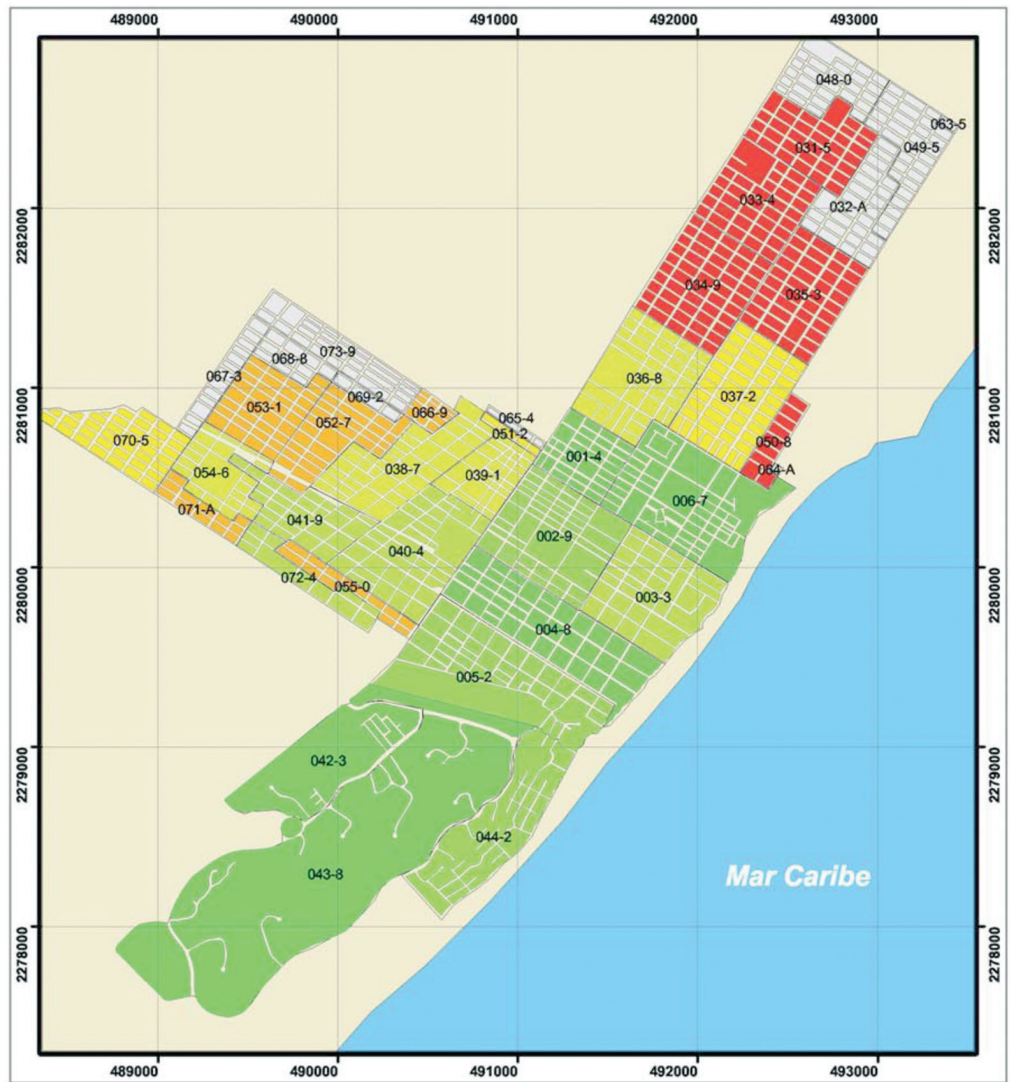

\section{Indicador Clave 6: Conexiones domiciliarias}

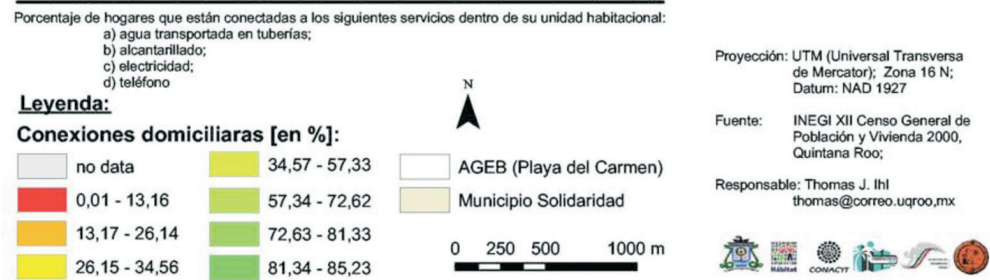

2-A. Playa del Carmen, 2000 


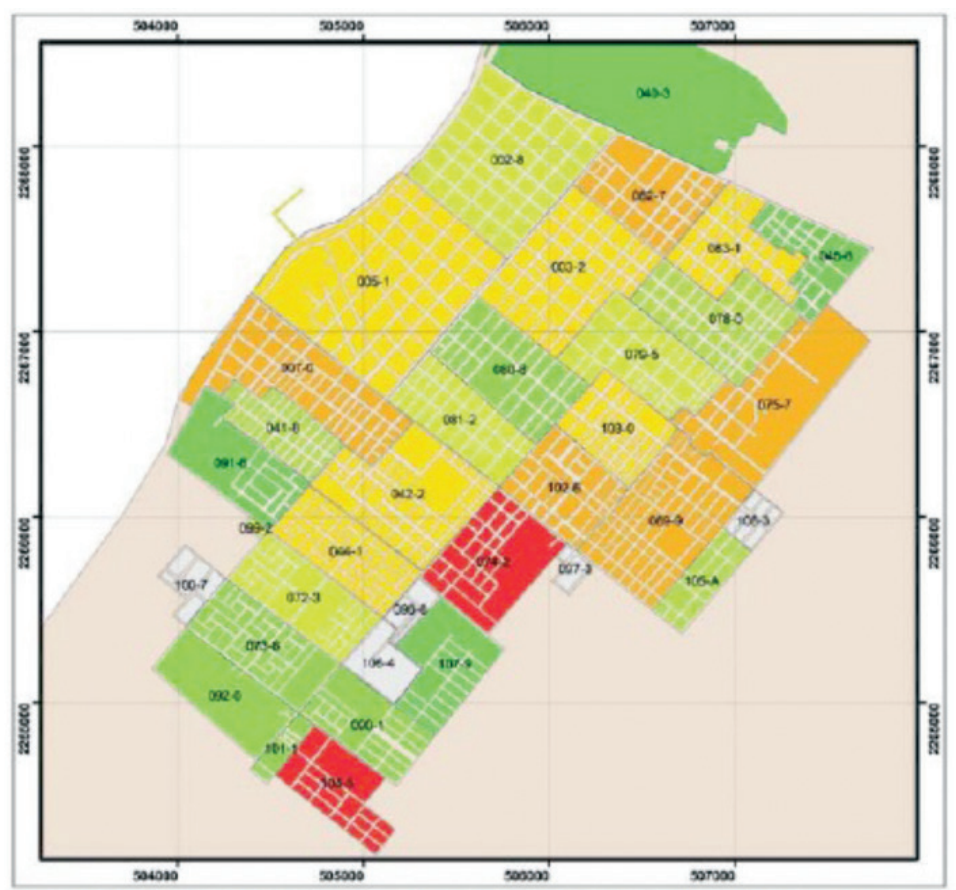

\section{Indicador Clave 6. Conexiones domiciliarias}

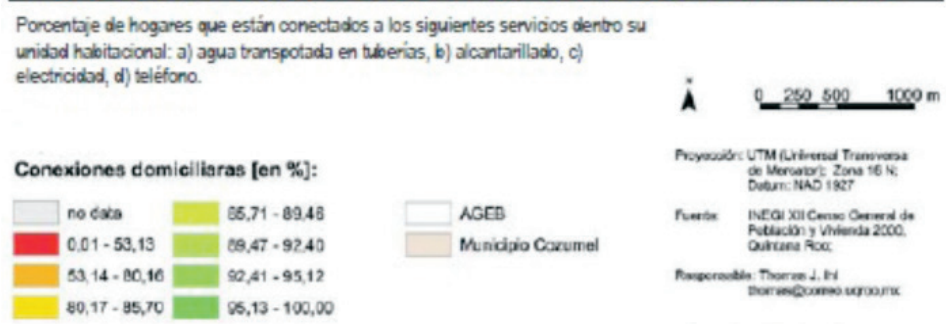

2-B. Cozumel, 2000

FIGURA 2-B. Distribución de Conexiones domiciliarias por AGEB en 2000 y 2010 


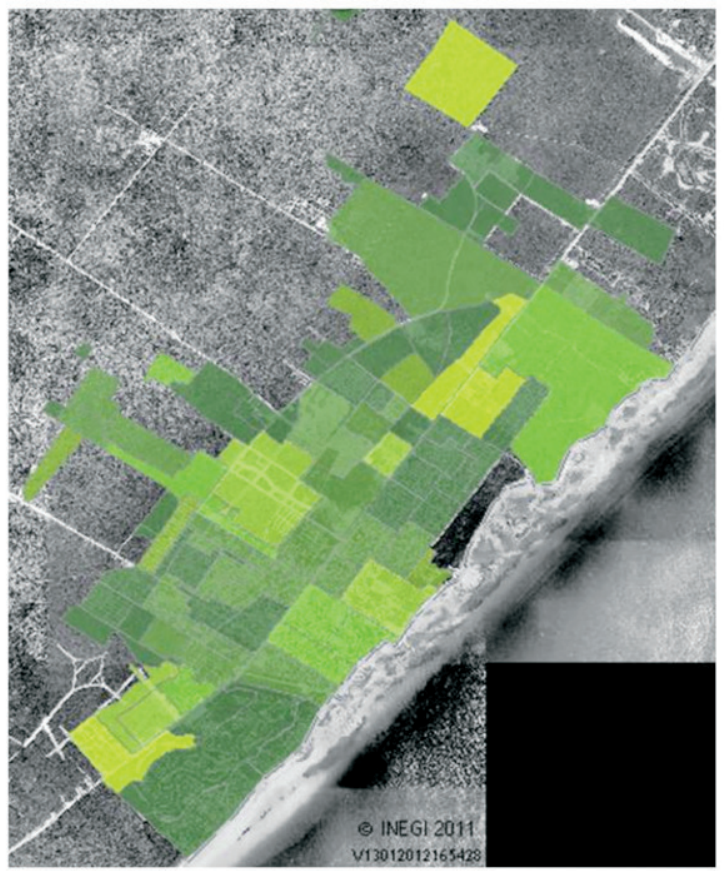

Indicador dave. Conexiones dorniciliarias por AGEB 2010 de la zona urbana de Playa del Carmen

\begin{tabular}{|c|c|c|}
\hline \multicolumn{3}{|c|}{ Conexiones domiciliarias } \\
\hline 司 & 95.21 & $c=100$ \\
\hline ? & 85.230 & $c=95.21$ \\
\hline ?.? & 81.33 & $<=85.23$ \\
\hline 7.) & 72.62 & $c=81.33$ \\
\hline . 2 & 57.33 & $<=72.62$ \\
\hline i. 3 & 3456 & $e=57.33$ \\
\hline .? & 26.14 & $<=34.56$ \\
\hline ?. & 13.16 & $c=26.14$ \\
\hline ple & $=0.01$ & $\Leftrightarrow 13.16$ \\
\hline
\end{tabular}

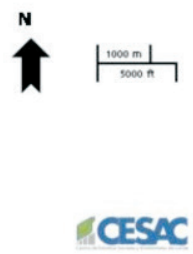

2-C. Playa del Carmen, 2010 


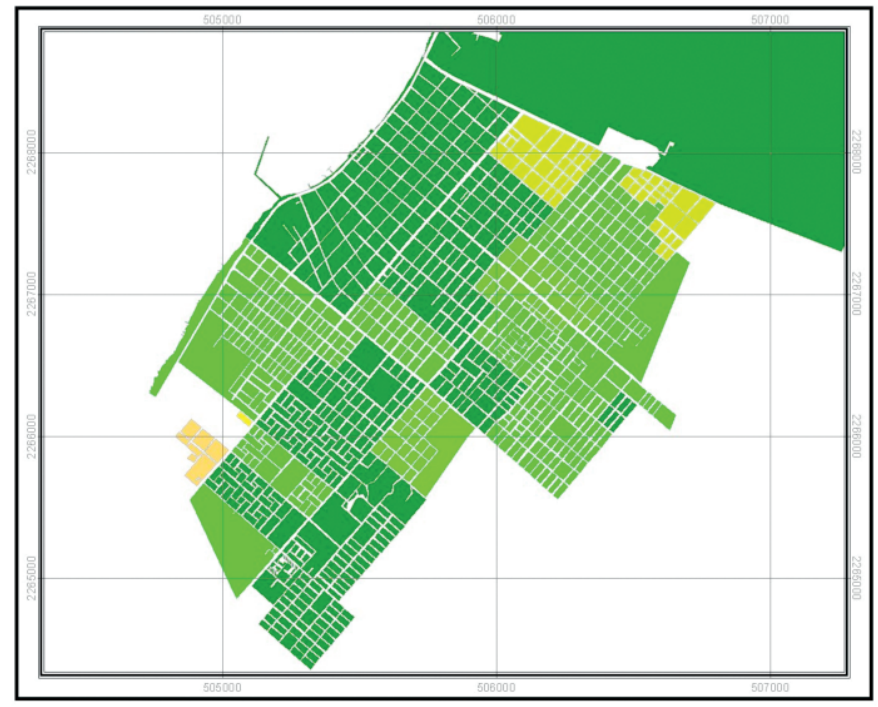

Indicador clave 6. Conexiones domiciliarias por AGEB 2010 de la zona urbana de Cozumel

Conexiones domiciliarias

\begin{tabular}{|l|l|}
\hline $0.01-53.13$ & $89.47-92.4$ \\
\hline $53.14-80.16$ & $92.41-95.12$ \\
\hline $80.17-85.7$ & $95.13-100$ \\
\hline $85.71-89.46$ & \\
\hline
\end{tabular}

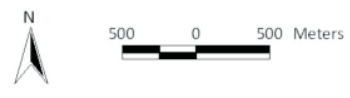

CESAC

2-D. Cozumel, 2010

FIGURA 2-D. Distribución de Conexiones domiciliarias por AGEB en 2000 y 2010 
En cambio, para ese mismo año en Cozumel (figura 2-B) no existen zonas claras donde se concentren las AGEB con las más bajas provisiones de este servicio, como sucedía en Playa del Carmen; se advierte más bien una perspectiva de mosaico, en la que se mezclan AGEB con altos y bajos porcentajes de provisión (figura 2).

No obstante esta mayor dispersión de los rangos de provisión del servicio, es posible identificar algunas características clave. Las AGEB con los porcentaje más bajos, entre 40 y $53.13 \%$, se ubican al sureste de la ciudad, aunque sin continuidad espacial, y corresponden a dos colonias: Стм y Maravilla, que están entre las de más reciente creación. También se aprecia que al este de la ciudad, en las colonias San Gervasio, Chen Tuk y Repobladores, esencialmente constituidas para viviendas de alta densidad, existen tres AGEB entre 53.14 y $80.16 \%$ de provisión -el segundo rango más bajo-, que se concatenan para formar una franja de AGEB con bajo desempeño de este indicador. Además de éstas se hallan otras aglomeraciones de AGEB en el mismo rango de provisión que muestran continuidad espacial: las AGEB 005-1, 003-2 y 0063-8, con grado de provisión de entre 80.17 y $85.70 \%$, las cuales inician en el centro de la ciudad y se desplazan al norte de forma escalonada; las AGEB 066-1 y 062-2, en el centro-sur de la ciudad, en las colonias Independencia y San Miguel 1, que están en un rango de entre 80.17 y $85.70 \%$; y en la parte sur de la ciudad las AGEB 092-0, 101-1, 073-6 y 000-1, que se localizan en las colonias San Miguel 2, Golondrinas, Base Militar y Flamingos, ubicadas en el rango de entre 92.41 y $95.12 \%$, por lo que pueden considerarse en buen nivel de provisión.

Para Playa del Carmen, las AGEB tienen en general porcentajes entre los niveles medios y altos con referencia al año 2000, lo cual conforma un panorama de buena provisión de estos servicios en las zonas de la ciudad. En cuanto a las AGEB con los porcentajes en los niveles medios, se localizan en el centro histórico de la ciudad, en el este de la ciudad en las colonias Ejidales, en la parte norte en la sección este de la colonia Luis Donaldo Colosio y la adyacente parte oeste de las colonias Mundo Hábitat y Bosque Real; así como en Villas Riviera, La Guadalupana y El Petén, en el sur en las AGEB de la colonia Campestre y sus alrededores es claro que están en la peores condiciones, como sucedió en las mediciones para el agua potable, por lo que serían las zonas con menor acceso a los servicios relacionados con el agua y su saneamiento en la zona urbana de Playa del Carmen.

Por su parte, Cozumel tiene también una mayoría de sus AGEB en niveles de medios a altos, sin embargo, en esta ciudad se observa una AGEB en el segundo 
menor rango de acceso a este servicio, la cual se ubica en la parte sur de la ciudad, en la sección sur de la colonia Colonos Cuzamil. Son dos las AGEB con porcentajes medios y se localizan al norte de la ciudad: una al norte de la colonia 10 de Abril y la otra al norte de la colonia Emiliano Zapata. Cabe apuntar que estas AGEB mostraron los porcentajes más bajos en el indicador de agua potable, por lo tanto, pueden considerarse entre las zonas con menor acceso a los servicios relacionados con el agua y su saneamiento en Cozumel.

\section{Discusión con el crecimiento poblacional urbano}

En esta sección se contraponen los resultados anteriormente expuestos con el indicador de crecimiento de promedio anual; el propósito es verificar una posible influencia del crecimiento poblacional en los movimientos de los indicadores estudiados.

CUADRO 4. Crecimiento poblacional medio anual 1995-2010

\begin{tabular}{|lccc|}
\multicolumn{1}{c}{ Ciudad } & \multicolumn{3}{c|}{ Periodo } \\
& $\mathbf{1 9 9 5 - 2 0 0 0}$ & $\mathbf{2 0 0 0 - 2 0 0 5}$ & $\mathbf{2 0 0 5 - 2 0 1 0}$ \\
\hline Cozumel (Cozumel) & 4.36 & 3.81 & 1.68 \\
Playa del Carmen (Solidaridad) & 19.87 & 18.14 & 3.29 \\
\hline
\end{tabular}

Fuente: Elaboración propia con base en el INEGI (2000, 2005 y 2010).

Es notorio (cuadro 4) que, respecto al indicador "Acceso al agua segura”, las dos ciudades inician con porcentajes sobre $90 \%$, pero el de Cozumel es ligeramente superior; aunque vienen, en el quinquenio de 1995 a 2000, de dos crecimientos poblacionales medios anuales a ritmos diferentes, donde el de Playa del Carmen es cuatro veces mayor que el de Cozumel (19.87 y $4.36 \%$, respectivamente). Ahora, como se mencionó en el periodo de 2000 a 2005, los porcentajes de este indicador se elevan en ambas ciudades ( 0.81 y 1.13 \% medio anual para Cozumel y Playa del Carmen, respectivamente), pero las poblaciones también crecen, aunque en distintas proporciones: $3.81 \%$ para Cozumel y $18.14 \%$ para Playa del Carmen. Si bien 
en términos reales los porcentajes en este indicador son superiores en Cozumel (99.54\%), es claro que los esfuerzos debieron ser mucho mayores para que Playa del Carmen llegara al porcentaje de provisión de 2005 (97.94\%). Posteriormente, en el lustro de 2005 a 2010, este indicador disminuye su ritmo de provisión en las dos ciudades: en Cozumel $-0.71 \%$, es decir decrece, mientras que en Playa del Carmen $0.20 \%$; en contraste, sus crecimientos poblacionales, aunque también son menores, permanecen en porcentajes sobre $1 \%$ (1.68 \% para Cozumel y $3.29 \%$ para Playa del Carmen), lo cual señalaría que a pesar de que en este quinquenio las ciudades tienen una menor presión poblacional, y podrían lograr más fácilmente una mayor cobertura de su provisión, en realidad, en términos proporcionales, su suministro ha mermado.

Respecto al indicador "Saneamiento mejorado", las ciudades comienzan la década con una gran diferencia en el suministro: Cozumel (87.42\%) con casi el doble de porcentaje que Playa del Carmen (45.37\%), lo cual, contrastado con el crecimiento poblacional de 1995 a 2000, muestra que Playa del Carmen tenía un crecimiento muy superior al de Cozumel -cerca de cinco veces más-, lo que señala que Playa del Carmen venía de mucho mayores presiones poblacionales y que, a diferencia del agua segura, posiblemente éstas pudieron influir en la baja cobertura inicial. En el primer quinquenio de la década se observa que ambas ciudades mejoran su provisión de saneamiento, sobre todo Playa del Carmen (5.97\% promedio anual, y Cozumel $2.27 \%$ ), y que sus crecimientos, aunque ligeramente menores al lustro anterior, aún muestran, especialmente en Playa del Carmen, signos de altos niveles (Cozumel 3.81 \% y Playa del Carmen 18.14 \%), lo cual apuntaría que a pesar de que en esta primera parte de la década Playa del Carmen tiene mucha mayor presión poblacional que Cozumel, logra mejorar en su cobertura hasta sobrepasar $60 \%$ de sus habitantes, pero no con la eficiencia alcanzada en el indicador de agua segura. En la segunda mitad de la década se advierte que los crecimientos poblacionales de ambas ciudades descienden de forma considerable: en Cozumel cerca de la mitad y en Playa del Carmen seis veces menos; en cambio, los niveles del indicador de saneamiento tienen movimiento dispares: en Cozumel disminuye ligeramente $-0.21 \%$ anual y en Playa del Carmen aumenta $10.03 \%$ anual. Esto sugiere que es muy probable que en el momento en que Playa del Carmen disminuye su crecimiento poblacional logra aumentar la provisión de este servicio, mientras que en Cozumel los datos refieren que al moverse muy poco la población tampoco habría sido necesario elevar el sumi- 
nistro de este indicador.

Por último, el indicador "Conexiones domiciliarias" manifiesta que al principio de la década se tiene, como en el anterior, una situación similar: provisiones menores a la mitad de los habitantes en Playa del Carmen e inferiores a $90 \%$ en Cozumel, en tanto que los crecimientos poblacionales de 1995 a 2000 muestran ser los más altos en todo el periodo de estudio. En cambio, en los primeros cinco años de la década este indicador crece en ambas ciudades, siempre Playa del Carmen en una mayor media anual (5.08\%) que Cozumel (1.87\%); asimismo, se presentan altos crecimientos poblacionales, que en el caso de Playa del Carmen son seis veces superiores a los de Cozumel. Se podría aseverar que en esta primera década la ciudad crece en su población a un ritmo que el crecimiento del indicador es incapaz de igualar. Esto explicaría por qué en 2005 el indicador apenas cubre 1 de cada 2 habitantes, mientras la población es casi $20 \%$ mayor cada año. En el segundo quinquenio se aprecia que, frente al alto crecimiento medio anual de este indicador en Playa del Carmen, la población disminuye considerablemente su ritmo de crecimiento, en tanto que en Cozumel aumenta ligeramente la provisión de este servicio, a la vez que aumenta ligeramente su población.

\section{Conclusiones}

El análisis realizado hasta aquí ha permitido evaluar, en dos localidades altamente presionadas por sus crecimientos poblacionales, los avances en la provisión de servicios básicos como saneamiento y agua potable, que son parte de la agenda mundial por el desarrollo. Entre los resultados obtenidos más relevantes están los siguientes:

1. En la mayoría de los casos, las ciudades estudiadas inician con porcentajes disparejos, aunque Cozumel siempre en una mejor situación que Playa del Carmen; sin embargo, al avanzar en el tiempo, la segunda ciudad acelera su provisión en mucho mayor medida que la primera, y así termina por acercársele o sobrepasarla.

2. Se presentan grandes crecimientos en los suministros de estos servicios básicos, es decir, mejora su provisión; sin embargo, al final de la década es notoria, sobre todo en Cozumel, una desaceleración de ese crecimiento, e 
incluso hay descensos de sus niveles.

3. Existen diferencias significativas en el abastecimiento de estos servicios básicos en el interior de las ciudades. Los datos indican que hay, por un lado, áreas con proporciones evidentemente inferiores a los resultados globales, que en mayor medida se ubican en las zonas populares y periféricas de las ciudades; y, por el otro, áreas con porcentajes arriba del promedio, generalmente situadas en las zonas centrales o residenciales. Al igual, es evidente que las ciudades presentan diferentes aglomeraciones de las carencias: Playa del Carmen con una alta concentración de las áreas con dificultades de provisión en ciertos sectores de la ciudad, y Cozumel, aunque tiene zonas con concentración de áreas, muestra una mayor dispersión en la mancha urbana.

4. Las presiones poblacionales parecen tener distintos efectos en la provisión de los indicadores y en diferentes momentos. Un elemento claro es que a pesar de los crecimientos poblacionales intensos las ciudades consiguen aumentar sus suministros. Cabe destacar que no obstante Playa del Carmen enfrenta los más altos crecimientos demográficos logra cubrir a la mayoría de los nuevos habitantes al grado de que se acerca o sobrepasa a Cozumel, que ha crecido en menor medida.

\section{FUENTES CONSULTADAS}

Boltvinik, J. (1999). “Conceptos y medidas de pobreza”, en J. Boltvinik y E. Hernández Laos. Pobreza y distribución del ingreso en México. México: Siglo XXI Editores, 30-80.

CIAMA (1992). Declaración de Dublín sobre el Agua y el Desarrollo Sostenible. Dublín: Conferencia Internacional sobre el Agua y el Medio Ambiente.

Conapo (2004). Seis ciudades alcanzarán el millón de habitantes entre 2000 y 2030. México: Consejo Nacional de Población.

------ (2009). Índice de marginación urbana 2005. México: Consejo Nacional de Población.

Coneval (2009). Metodología para la medición multidimensional de la pobreza en 
México. México: Consejo Nacional de Evaluación de la Política de Desarrollo Social.

INEGI (1996). Conteo de Población y Vivienda 1995. México: Instituto Nacional de Estadística y Geografía.

---- (2001). XII Censo General de Población y Vivienda 2000. México: Instituto Nacional de Estadística y Geografía.

---- (2006). II Conteo de Población y Vivienda 2005. México: Instituto Nacional de Estadística y Geografía.

---- (2010). Estadísticas a propósito del Día Mundial de la Población. Datos de Quintana Roo. México: Instituto Nacional de Estadística y Geografía.

---- (2011). Censo de Población y Vivienda 2010. México: Instituto Nacional de Estadística y Geografía.

Lavell, A. (2000). “Desastres urbanos: una visión global”. La Red [en línea]. Bogotá:

Red de Estudios Sociales en Prevención de Desastres en América Latina. Disponible en: http://www.desenredando.org/public/articulos/2000/duuvg/ index.html [2011, 14 de octubre].

ONU (2000). Declaración del Milenio. Nueva York: Asamblea General de las Naciones Unidas.

--- (2002). Informe de la Cumbre Mundial sobre el Desarrollo Sostenible. Nueva York: Organización de las Naciones Unidas.

--- (2010a). El derecho humano al agua y el saneamiento. Nueva York: Asamblea General de las Naciones Unidas.

--- (2010b). The Right to Water. Ginebra: Office of the United Nations High Commissioner for Human Rights (Fact Sheet No. 35).

ONU-Hábitat/Sedesol (2012). Guía metodológica para la constitución y operación de las Agencias de Desarrollo Urbano y los Observatorios Urbanos Locales. México: Programa de las Naciones Unidas para los Asentamientos Humanos/Dirección General de Desarrollo Urbano y Suelo, Secretaría de Desarrollo Social.

Rojas López, J. (2010). "Vulnerabilidad socioambiental a la influenza tipo A (H1N1) en la Península de Yucatán, México”, en O. Frausto Martínez (coord.). Desastres e impactos socioambientales provocados por el virus de la influenza humana A (H1N1): experiencias compartidas México-Costa Rica. México: Universidad de Quintana Roo/Universidad de Costa Rica. 